\title{
A review on sparse Fast Fourier Transform applications in image processing
}

\author{
Hadhrami Ab. Ghani ${ }^{1}$, Mohamad Razwan Abdul Malek ${ }^{2}$, Muhammad Fadzli Kamarul Azmi ${ }^{3}$, \\ Muhammad Jefri Muril ${ }^{4}$, Azizul Azizan ${ }^{5}$ \\ 1,2, 3, ${ }^{4}$ Faculty of Engineering and Technology, Multimedia University, Malaysia \\ ${ }^{5}$ Advanced Informatics School, Universiti Teknologi Malaysia, Malaysia
}

\begin{tabular}{l} 
Article Info \\
\hline Article history: \\
Received May 3, 2019 \\
Revised Oct 14, 2019 \\
Accepted Oct 25, 2019 \\
\hline
\end{tabular}

Keywords:

Applications

Computational complexity

Fourier transform

Image processing

\begin{abstract}
Fast Fourier Transform has long been established as an essential tool in signal processing. To address the computational issues while helping the analysis work for multi-dimensional signals in image processing, sparse Fast Fourier Transform model is reviewed here when applied in different applications such as lithography optimization, cancer detection, evolutionary arts and wasterwater treatment. As the demand for higher dimensional signals in various applications especially multimedia appplications, the need for sparse Fast Fourier Transform grows higher.
\end{abstract} All rights reserved.

\section{Corresponding Author:}

Hadhrami Ab. Ghani,

Faculty of Engineering and Technology,

Multimedia University,

Jalan Ayer Keroh Lama, 75450 Bukit Beruang, Melaka, Malaysia.

Email: hadhrami.abdghani@mmu.edu.my

\section{INTRODUCTION}

Fourier Transform is first introduced by Jean Baptiste Joseph Fourier [1] to solve the computational complexity in wide varities of fields including earth and science, chemistry, communications, and signal processing [2-5]. In signal processing, Fourier Transform [6-11] has long been established as an instrumental tool applied in electrical signal spectrum and filter analysis, sampling and series, antenna, television image convolution as well as radio broadcasting [1]. Being the limiting case of Fourier Series for non-periodic signals, FT is used to convert signal to frequency domain as the frequency domain has many superlative benefits especially for analytical purposes rather than in the classical time domain. In order to solve different various problems especially in digital image processing, the disceret version of FT, typically regarded as Discrete Fourier Transform (DFT) has been formulated.

Due to the large number of discrete samples required for DFT operation which requires $N^{2}$ complex multiplications, Fast Fourier Transform (FFT) has been introduced to significantly reduce the computational complexity by just requiring $N \log N$ multiplications for $\mathrm{N}$ samples. This computational complexity issue becomes worse when higher dimensional signals such as image signals, which are two dimensional, are represented and processed using FFT. To further reduce the computational complexity, a sparse FFT model has been proposed where the number $\mathrm{k}$ of coefficients used in the FFT operation is less than $\mathrm{N}$ [12]. This proposed sparse FFT model is also motivated by the fact that many real signals in practice are approximately sparse, having only a number of relatively large and nonzero signal elements as compared with the rest of the signal components. Therefore to save the computational time and reduce its complexity, the approximately zero signal components will not be measured in the sFFT operation. Hence, this paper is 
written to address this issue by giving a concise review on the applications of sparse FFT in different applications in image processing.

\section{LITERATURE REVIEW}

FFT is a useful signal representation method that can be applied for fast processing especially when the signals are two dimensional like image signals. The image signals can be transformed using FFT in image processing since the signals are typically structured, rendering a sparse spectrum [13]. Although FFT is known to has reduced computational complexity than DFT, further simplication is possible by representing this signal by using only $\mathrm{k}$ coefficients, assuming that this signal of length $\mathrm{N}$ is a $\mathrm{k}$-sparse $(\mathrm{k}<\mathrm{N})$ signal. Based on this finding, signal compression is possible and has been implemented in various compression algorithms such as MP3 [14]. As the number $\mathrm{k}$ of the coefficients is now less than N, it is straightforward to know that the FT operations can be further simplified with lower computational complexity.

Various FT algorithms have been proposed in literature to reduce the computational complexity, as presented in [15]. One of the approaches which have been widely referred to in literature is the sparse FFT(sFFT) presented in [15]. However this method is formulated for one-dimensional case only. Therefore signals which are two dimensional such as image signals are unable to be represented using a onedimensional sFFT model. The many image processing applications such as lithography, medical imaging, evolutionary arts and particle detection in wastewater treatment are infeasible to be applied with a one-dimensional sFTT.

A multi-dimensional sFFT has been proposed in [13] to cater the multi-dimensional cases. This multi-dimensional sFFT, including the 2D sFFT, has been widely studied and applied in a number of fields such as radar signal processing, lithography illumination and deep learning, which will be reviewed in this paper. From this point onwards, this paper is organized as follows. The general formulation of multi-dimensional sFFT is briefly described in Section 3. In Section 4.1 until 4.5, the applications of multi-dimensional sFFT in image processing will be studied and reviewed. A brief account on the challenges and future work related to sFFT applications in image processing will be given in Section 5 before the paper is concluded in Section 6.

\section{MULTI-DIMENSIONAL SPARSE FAST FOURIER TRANSFORM (sFFT)}

The fundamental idea in sFFT for reducing the computational complexity is to consider only $k$ nonzero coefficients, out of $N$ coefficients of the signal. For $M$-dimensional signal $x_{i_{1}, i_{2}, \cdots, i_{M}}$ where $x \in$ $\mathbb{C}^{N_{1} \times N_{2} \times \cdots \times N_{M}}$ and $\omega_{m}=e^{2 \pi i / N_{m}}$, the FT signal $\hat{x}_{i_{1}, i_{2}, \cdots, i_{M}}$ can be expressed as

$$
\hat{x}_{i_{1}, i_{2}, \cdots, i_{M}}=\sum_{j_{1}}^{N_{1}} \sum_{j_{2}}^{N_{2}} \cdots \sum_{j_{M}}^{N_{M}} \omega_{1}^{i_{1} j_{1}} \omega_{2}^{i_{2} j_{2}} \cdots \omega_{M}{ }^{i_{M} j_{M}} x_{j_{1}, j_{2}, \cdots, j_{M}}
$$

Without FFT, the computational complexity required in the above equation is very high, which is $\mathrm{O}\left(N_{1} N_{2} \ldots N_{M}\right)$, hence impractical and time-consuming. With FFT, the computational complexity is significantly reduced. However it is very common in practice to have sparse signals which have only a number of non-zero components. In other words some of the components of the signal, which are zero, can be excluded from the Fourier Transform calculation to further reduce the computational complexity and processing time.

This computational load reduction is especially important for signals with significantly large numbers of components and high dimensionality. Therefore multidimensional sparse FFT technique has been proposed in different forms in literature to address varying research problems. The main idea of sparse FFT technique is to identify the sparse components, which are typically more significant in other small or closedto-zero components of the signal which are excluded in the sparse FFT operation to reduce the computational load. Sparse signals are very common in nature, as the signal components tend to have varying amplitude values some of which are relatively larger than the other signal components. In the next section, the applications of multidimensional sparse FFT will be discussed.

\section{APPLICATIONS OF MULTI-DIMENSIONAL SFFT}

As the number of components for multi-dimensional signals is huge, finding the peaks of the signal components requires relatively high computational complexity. The commonly applied method in literature for finding the peaks is termed as spectrum permutation, which has been demonstrated in [16] that in order to optimize the spectrum permutation, the permutation parameters must be carefully selected. Random selection 
of the permutation parameters may not guarantee the optimized solution. To obtain the optimized permutation parameters, lattice theory is applied. Pseudorandom approach is also applicable to obtain the optimized parameters.

\subsection{Optimization of lithography source illumination}

Lithography is a kind of printing methods which has been introduced many years ago starting primarily with the use of lithographic stones to print texts or images on smooth surfaces. Nowadays, lithography is widely used for high-volume printing including books, newspapers and posters. In electronics, lithography is used for high-volume fabrications of integrated circuits [17, 18], which face the imaging distortion problem when the critical dimension of the target integrated circuits reduces. The effective method to improve the quality of the image in the lithography process is via lithography illmuniation optimization (ILO). As the required computational complexity for calculating the sparse mask layout during the lithography process is very high, the sFFT approach has been adopted to reduce the computational complexity and render a more robust approach. This approach [17] applies a compressive sensing method that focusses only on the pixels located in the optimized subspace, which contains pixels that cause interference. By using the sFFT, the ILO process which is based on the CS method uses a relatively small number of variables, rendering significantly reduced computational complexity and improved computational speed, in addition to the improved quality of the ILO image.

\subsection{Image evolution and perceived characteritics measurement}

In image processing, one of the actively explored fields is procedural generation [19, 20], which applies algorithms to generate evolutionary art and images such as textures and multi-dimensional images. As the procedurally generated images tend to be random in nature, heuristic approachs have been employed to measure some of the perceived features of the images such that better improvements are feasible to be implemented in generating the images [21]. Identifying the cognitive perceived features of digital images is challenging. An effective measurement of these perceived features is the use of spatial frequencies, as proposed in [21]. The power spectral density, which can be determined via the sFFT operation, can be computed from the spatial frequencies for image retrieval or classification purposes. By using the spectrum obtained via the multi-dimensional sFFT operation, the target images can be generated and synthesized such that they share the same spectral properties. The strategies applied to generate these imageas can be also adjusted according to the target types of images such as greyscale and colour images.

\subsection{Radar signal processing}

A radar system, which is typically employed to detect moving objects, applies the concept of Doppler phenomenon. As the calculation of the position of the moving object is real time and needs to be updated instantaneously, the high computational complexity required to process the continuous wave radar signal can be reduced using sFFT. With just a number of significant frequency components, sFFT has a relatively low computational complexity and suitable to be implemented in short-range ubiquitous radar signal processing [16]. With a slight improvement in the sFFT approach proposed in [13], an improved sFFT model has been designed such that it is more robust to the effects of noise. Furthermore, Neyman-Pearson detection approach is also incorporated in [16] in order to reduce the required computational complexity in finding the exact sparsity when operating the sFFT approach.

\subsection{Fast nonlinear compressive sensing lithographic source}

Another important research field in lithography [22] which applies Fourier Transform is the source and mask optimization (SMO), which is essential in improving the reliability of the lithography imaging operation [23]. However one the main challenges of applying SMO is the high computational complexity due to the inverse reconstruction problem which is nonlinear. A compressive sensing (CS) theory has been implemnted in [24] to reduce the high computational load in SMO, which limits its applications to chip-level applications. The sFFT is suitable to represent the sparse signals in the mask and source patterns sampled from the SMO operation. The nonlinear nature of CS theory can be used to formulate the SMO using the sFFT as the source and mask patterns are typically sparse in nature.

\subsection{Identification of microplastic in waste water treatment plants}

Protecting the environment constitutes as one of the major principles in engineering. The wastewater produced by the many factories, without proper treatment, will pollute the water and land. Any polymer particles which are smaller than $5 \mathrm{~mm}$ in length are classified as microplastic [25], which can be easily found in cosmetics products. Microplastic ruins our natural ecosystem besides polluting the environment especially the river and ocean as microplastic fails to dissolve in water. As reported in [26, 27], the amount of 
microplastic in the river and ocean has increased and affected the ecosystem. In order to treat the polluted water, the intensity of the polluted water must be measured. However, calculating the intensity of the water is challenging due to the small size of microplastic, which is hard to be identified in the watter. In order to improve the measurement, a focal plane array method which is designed based on FT has been proposed to measure the intensity of the water [25]. It has been reported that this method is able to detect very small microplastic particles, down to $20 \mathrm{~mm}$ in length. Therefore more accurate results have been obtained and faster measurement time is also possible with the implementation of FT method.

\subsection{Cancer detection}

Cancer is one of the most critical diseases that have taken many lives. Cancer starts from a mutation cell that possesses irreversible and transmittable genetic abnormalities with the presence of epigenetic variation in cells [28]. Cancer cells are able to quickly grow in number subject to the microenvironment of the cells. A commonly applied treatment is the chemotherapy which attempts to kill the cancer cells to stop them from growing and infecting other parts of the body. Since cancer is considered as a dangerous illness, early detection of this disease is highly encouraged. One of the early signs of cancer is the growth of tumor. The typical procedures to diagnose cancer are oftern expensive and require a number of phases to confirm the disease. Therefore reducing the time required for the diagnosis procedures is highly preferable and needed. The common diagnosis procedure is known as histopathology which requires a conciderably long time to verify the result after taking tissue samples of the patient. Fourier Transform Infrared spectroscopy (IR) $[29,30]$ method observes the vibrational spectral analysis of the structural components of the cells such as the intensity, band shape, frequency and band splitting. This method is non-destructive as it detects any changes in the molecules of the functional groups in the sample tissues or cells being visualised in mid infrared region. Furthermore only a small quantity of samples is needed to perform this test, with a fast analysis of biochemical variations at molecular level. An FT-based spectroscopy method is applied to perform deep analysis regarding the structural of the cells. This method is also capable of differentiating between unaffected and magliant tissues by comparing the changes of $\mathrm{C}-\mathrm{O}$ and $\mathrm{CH}$ molecules in vibrationing modes.

\section{CHALLENGES AND FUTURE WORKS}

As the need for higher dimensional signal increases, the applications of sFFT are expected to become more relevant and important. The fast progress of telecommunication industry in providing larger bandwith with increasing data speed will further encourage the research activities in image processing. However as the demand increases for these high speed applications, the bandwidth allocation must be fairly managed. As the bandwith increases, a larger number of signal components will need to be represented. Such situations will definitely need the sparse FFT model so as to reduce the potentially large computational complexity when representing the signals having large bandwidth. This is one of the major challenges in the applications of sFFT in future. It is expected to be more challenging when higher dimensional signals such as the image signals are represented and processed. Dealing with higher dimensional signals, which are highly demanded by the users in this age for using especially multimedia applications, are real and hence must be addressed and solved.

\section{CONCLUSION}

In this report, the applications of the sparse Fourier Transform in image processing are reviewed. With the sparse signals found in different areas such as lithography, cancer detection, wastewater treatment and evolutionary arts, sparse Fast Fourier Transform has been demonstrated to be applicable to be integrated as the method to tackle issues in the aforementioned applications. In summary, it can be observed that sparse Fourier Transform is useful in reducing the required computational complexity especially when the signals to be represented are sparse, as seen in the cases of lithography optimization, cancer detection and image generation in evolutionary arts. Furthermore, in some applications like the wasterwater treatment of microplastic particles, it has been demonstrated that the sparse Fourier Transform application is capable of producing more accurate measurement of the polluted water intensity.

\section{ACKNOWLEDGEMENTS}

The authors acknowledge and thank all the supports especially from the Faculty of Engineering and Technology, Multimedia University, as well as all individuals who are directly or indirectly involved in preparing this paper. 


\section{REFERENCES}

[1] Boehme TK, Bracewell R. "The fourier transform and its applications”, Am Math Mon. 2006.

[2] Latfaoui M, Bereksi Reguig F. "Packets wavelets and stockwell transform analysis of femoral Doppler ultrasound signals", International Journal of Electrical and Computer Engineering (IJECE), Vol. 8, No. 6, pp. 4212-4220, December 2018.

[3] Jaber AA, Bicker R. "Development of a condition monitoring algorithm for industrial robots based on artificial intelligence and signal processing techniques", International Journal of Electrical and Computer Engineering (IJECE). Vol. 8, No. 2, pp. 996-1009, April 2018.

[4] Gurcan MK, Ab Ghani H. "Small-sized packet error rate reduction using coded parity packet approach", IEEE International Symposium on Personal, Indoor and Mobile Radio Communications, PIMRC. 2010.

[5] Albashier MAM, Abdaziz A, Ghani HA. "Performance analysis of physical layer security over different error correcting codes in wireless sensor networks", International Symposium on Wireless Personal Multimedia Communications, WPMC. p. 191-5, 2018.

[6] Ghani HA, Aziz AA, Azizan A, Daud SM. "Adaptive interference mitigation with user grouping for fast transmission in cellular networks", Indonesian Journal of Electrical Engineering and Computer Science, Vol. 10, No. 2, pp. 702-712, 2018;

[7] Abdul Aziz NH, Ab Ghani H, Syahali S, Hamzah MH. "Ant-colony algorithm with interference cancellation for cooperative transmission”, IET Signal Process. Vol. 10, No. 6, pp. 603-10, 2016.

[8] Nasser Hamad, Maen Takruri MB. "Wavelet based multicarrier CDMA system", International Journal of Electrical and Computer Engineering (IJECE). Vol. 9, No. 4, pp. 3051-3059, 2019.

[9] Sani ZM, Sen LW, Ghani HA, Besar R. "Real-time daytime road marker recognition using features vectors and neural network", 2015 IEEE Conf Sustain Util Dev Eng Technol CSUDET, pp. 38-43. 2016.

[10] Bin Hamzah MH, Bin Ab Ghani H. "Energy-limited ant colony optimization with cooperative transmission", I4CT 2014 - 1st Int Conf Comput Commun Control Technol Proc., PP. 161-5, 2014.

[11] Sani ZM, Ghani HA, Besar R, Loi WS. "Daytime road marker recognition using grayscale histogram and pixel values", Internetworking Indones J., Vol. 8, No. 1, pp. 11-6, 2016.

[12] Indyk P, Kapralov M, Price E. "(Nearly) Sample-Optimal Sparse Fourier Transform”. In 2013.

[13] Rauh A, Arce G. "Sparse 2D Fast Fourier Transform”, Proc 10th Int Conf Sampl Theory Appl. 2013.

[14] Musmann HG. "Genesis of the MP3 audio coding standard", IEEE Trans Consum Electron. 2006.

[15] Akavia A. "Deterministic sparse fourier approximation via approximating arithmetic progressions", IEEE Trans Inf Theory. 2014.

[16] Rauh A, Arce GR. "Optimized Spectrum Permutation for the Multidimensional Sparse FFT", IEEE Trans Signal Process. 2017.

[17] Ma X, Wang Z, Lin H, Li Y, Arce GR, Zhang L. "Optimization of lithography source illumination arrays using diffraction subspaces", Opt Express, 2018.

[18] Ma X, Song Z, Li Y, Arce GR. "Block-based mask optimization for optical lithography", Appl Opt., 2013.

[19] Freiknecht J, Effelsberg W. "A Survey on the Procedural Generation of Virtual Worlds", Multimodal Technol Interact, 2017.

[20] Cisneros RE, Wood K, Whatley S, Buccoli M, Zanoni M, Sarti A. "Virtual reality and choreographic practice: The potential for new creative methods", Body, Sp Technol. 2019.

[21] Gircys M, Ross BJ. "Image Evolution Using 2D Power Spectra”, Complexity, 2019;

[22] Ma X, Wang Z, Li Y, Arce GR, Dong L, Garcia-Frias J. "Fast optical proximity correction method based on nonlinear compressive sensing", Opt Express. 2018.

[23] Pirati A, Peeters R, Smith D, Lok S, van Noordenburg M, van Es R, et al. "EUV lithography performance for manufacturing: status and outlook", Extreme Ultraviolet (EUV) Lithography VII. 2016.

[24] Sun Y, Sheng N, Li T, Li Y, Li E, Wei P. "Fast nonlinear compressive sensing lithographic source and mask optimization method using Newton-IHTs algorithm", Opt Express, 2019.

[25] Sun J, Dai X, Wang Q, van Loosdrecht MCM, Ni BJ. "Microplastics in wastewater treatment plants: Detection, occurrence and removal", Water Research, 2019.

[26] Eerkes-Medrano D, Thompson RC, Aldridge DC. "Microplastics in freshwater systems: A review of the emerging threats, identification of knowledge gaps and prioritisation of research needs", Water Research, 2015.

[27] Li J, Liu H, Paul Chen J. "Microplastics in freshwater systems: A review on occurrence, environmental effects, and methods for microplastics detection", Water Research, 2018.

[28] Sahu RK, Mordechai S. "Fourier transform infrared spectroscopy in cancer detection", Future Oncology, 2005.

[29] Talari ACS, Martinez MAG, Movasaghi Z, Rehman S, Rehman IU. "Advances in Fourier transform infrared (FTIR) spectroscopy of biological tissues”, Applied Spectroscopy Reviews, 2017.

[30] Santos F, Magalhaes S, Henriques MC, Fardilha M, Nunes A. "Spectroscopic Features of Cancer Cells: FTIR Spectroscopy as a Tool for Early Diagnosis", Curr Metabolomics, 2018. 


\section{BIOGRAPHIES OF AUTHORS}
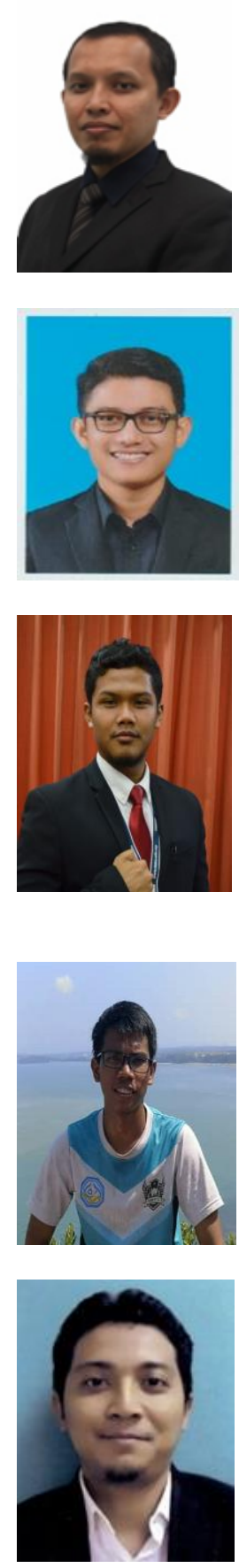

Hadhrami Ab Ghani received his bachelor degree in electronics engineering from Multimedia University Malaysia (MMU) in 2002. In 2004, he completed his masters degree in Telecommunication Engineering at The University of Melbourne. He then pursued his Ph.D. at Imperial College London in the same study area and completed his Ph.D. research in 2011. Currently, he serves as one of the academic and research staff members at MMU.

Mohamad Razwan Abdul Malek enrolled for Foundation in Engineering course at Multimedia University (MMU) in 2016. He had been awarded a full scholarship by Yayasan Telekom Malaysia to further his study at MMU. Currently he is in his third year and expected to finish his study before 2021 .

Muhammad Fadzli Kamarul Azmi began studying at Multimedia University (MMU) in 2016 before completing his study in 2017. He was one of the Yayasan Telekom Malaysia scholarship recipients studying at MMU. Currently he is pursuing his bachelor degree in Electronics Engineering majoring in Telecommmunications at MMU.

Muhammad Jefri Muril is Yayasan Telekom Malaysia's scholar who started his Foundation in Engineering study at Faculty of Engineering and Technology, Multimedia University (MMU) Malaysia in 2016. Currently he is in his third year of study, enrolling for a bacheloer degree in Electronics Engineering majoring in Telecommunications at MMU. His current CGPA is 3.88 out of 4.0.

Azizul Azizan obtained his PhD qualification (2009), from University of Surrey researching on 3.5G physical layer adaptation for satellite systems. He later joined the Malaysian Communication and Multimedia Commission for more than 6 years overseeing spectrum and numbering policies and resource management. He is currently with Advanced Informatics School (AIS), Universiti Teknologi Malaysia Kuala Lumpur where his research areas includes Telecommunications, Cyberphysical Systems (inc IoT), Business Intelligence and Engineering Education. 